\title{
Mechanical Properties of Evaporated Gold Films. Hard Substrate Effect Correction
}

\author{
Ke Du ${ }^{1}$, Xiaolu Pang ${ }^{1,2}$, Chi Chen ${ }^{1}$, and Alex A. Volinsky ${ }^{1}$ \\ ${ }^{1}$ Department of Mechanical Engineering, University of South Florida, 4202 E Fowler \\ Ave. ENB118, Tampa, FL, 33620 \\ ${ }^{2}$ Department of Material Physics and Chemistry, University of Science and Technology, \\ Beijing, Beijing, 100083, China, People's Republic of
}

\begin{abstract}
Nanoindentation tests using the Berkovich indenter tip were performed on 50 and $200 \mathrm{~nm}$ thick polycrystalline gold films deposited on hard substrates. Gold film hardness increased with the indentation depth due to the influence of the substrate. A procedure based on the Joslin-Oliver method was introduced to correct for the substrate effect. The method utilizes the fact that the measured elastic modulus did not change significantly with the indentation depth for the two films tested, which allowed reducing the contact area variation used in the hardness calculation.
\end{abstract}

\section{Introduction}

The ability to measure mechanical properties of thin films is important for various applications. Typically thin films have higher hardness compared with their bulk counterparts. There are many methods used for measuring thin films mechanical properties, which utilize different sample geometries, including freestanding films [1], microbridge [2], and microbeam cantilever beam [3] to name a few. However, nanoindentation is the most commonly used method for measuring mechanical properties. When indenting bulk polycrystalline samples their hardness and modulus do not change significantly with indentation depth, however, for thin films, the elastic modulus typically changes a lot at very shallow indentations $(<10 \%$ of the film thickness) and tends to flatten at deeper indentations. Hardness exhibits large scatter at very shallow depths, and then changes steadily with the indentation depth. For the very shallow indentations, the hardness variation can be explained by the indentation size effect or strain gradient plasticity theory [4]. Hardness change at deeper indentations can be explained by the substrate effect [5], although hardness, a measure of the materials ductile properties, should not change significantly with indentation depth. Some researchers argue that the increase in hardness may be caused by the indentations performed on the grain boundaries rather than the grains [6]. However, the same may also happen in bulk materials, where experimental data normally does not show such changes. Generally speaking, the hardness and elastic modulus of thin films are affected by the substrate. In order to correct the hardness, lots of models have been introduced. One of the most commonly used is the Oliver and Pharr method [7]. Oliver and Pharr used the elastic unloading slope of the load-displacement curve along with the calibrated tip area function to assess the elastic and plastic mechanical properties: 


$$
\begin{gathered}
\frac{1}{E_{r}}=\frac{\left(1-v_{i}^{2}\right)}{E_{i}}+\frac{\left(1-v_{f}^{2}\right)}{E_{f}} \\
E_{r O P}=\frac{\sqrt{\pi}}{2 \beta} \frac{S}{\sqrt{A_{c}}} \\
H_{O P}=\frac{P}{A_{c}}
\end{gathered}
$$

where $E_{r}$ is the reduced modulus, $\beta$ is an indenter shape constant, $S$ is the stiffness, $E_{i}$ and $E_{f}$ are the Young's moduli of the indenter and the film, $v_{i}$ and $v_{f}$ are the Possion's ratios of the indenter and the film materials, respectively. The Oliver-Pharr method was originally applied for the bulk materials and in the case of elastic mismatch between the film and the substrate can lead to errors. One of the main problems of the Oliver-Pharr method is that the pile-up and sink-in phenomena can not be accounted for. Soft ductile films (mostly metals) on hard substrates can exhibit significant pile-up. Figure 1a shows plastic pileup around the Berkovich tip indent into $200 \mathrm{~nm}$ gold film on mica. The pile-up diameter increases as a function of the maximum indentation load in Figure $1 b$.
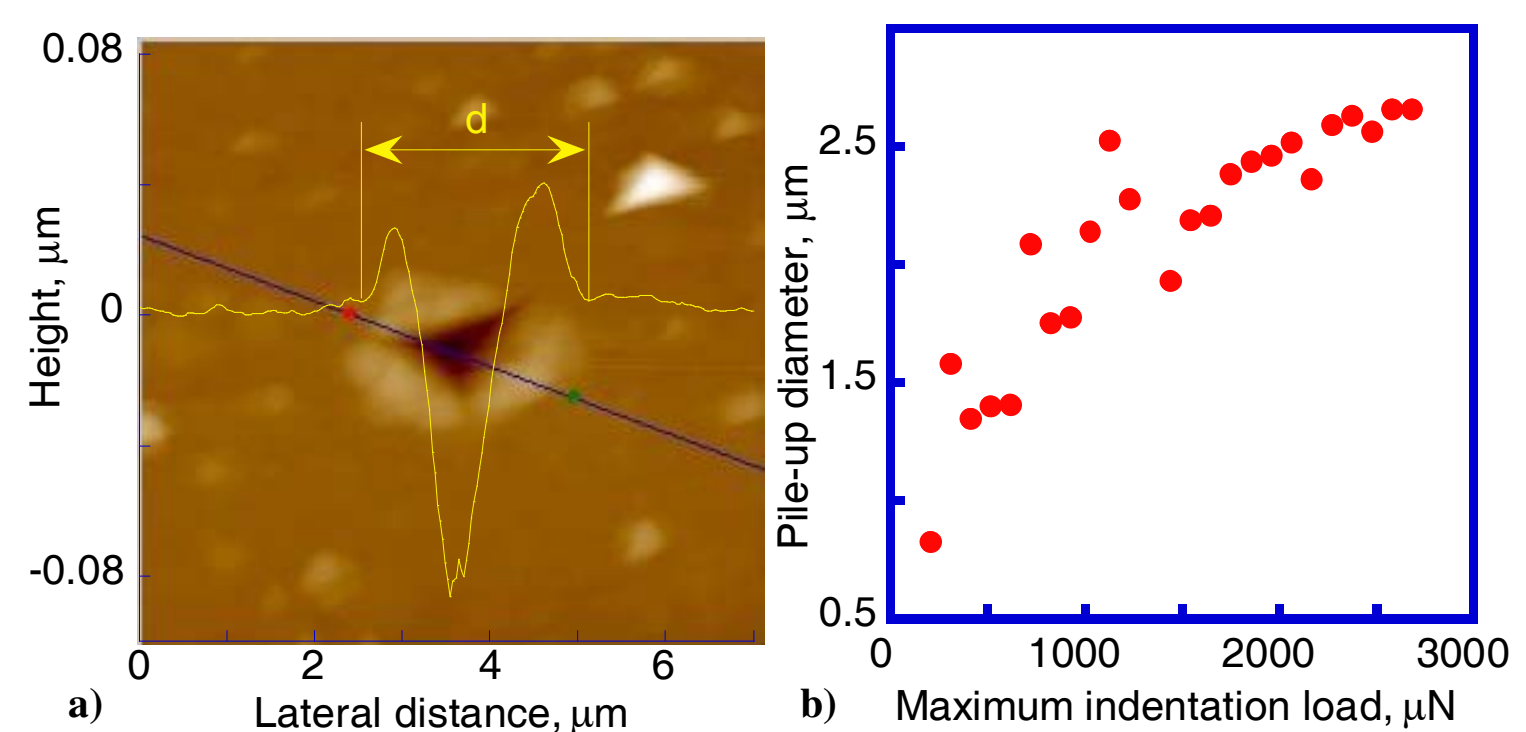

Figure 1. a) Pile-up around indentation of $200 \mathrm{~nm}$ gold film on mica with Berkovich tip; b) Plastic pile-up diameter as a function of the maximum indentation load.

For the hard films on soft substrates, the sink-in can be observed. Another problem with the Oliver-Pharr method is associated with the assumption that the film is smooth. However, the surface roughness may significantly affect the mechanical properties measurements. Many researchers have suggested that the indentation depth should be less than $10 \%$ of the film thickness. This approach can give satisfactory results for thick films, however, for very thin films (nanoscale), the surface roughness, machine 
compliance and indentation size effect can influence the results.

Considering all these problems, Joslin and Oliver [8] adopted another method that can minimize the surface roughness, pile-up and sink-in effects when calculating film hardness. In the Joslin-Oliver method, the contact area is eliminated. As a result, equations (2) and (3) can be rearranged as:

$$
H_{J O}=\frac{4 \beta^{2}}{\pi} \frac{P}{S^{2}} E_{r}^{2}
$$

In this method, reduced modulus, $E_{r}$, was assumed to be constant and not changing with the indentation depth. For soft films on hard substrates, the reduced modulus can increase with the indentation depth. Both the reduced modulus and the hardness measurements are influenced by the substrate. Joslin and Oliver [8] suggested that the ratio of the indentation load and the square of the unloading stiffness, $P / S^{2}$, is a material characteristic. For bulk materials, the value of $P / S^{2}$ does not change much with the indentation depth [9]. Former studies have shown that the Joslin-Oliver method is only good for homogeneous materials with similar modulus [9], thus many researchers utilized FEM to model the substrate effect and correct the measured reduced modulus. King et. al. [10] used a flat punch model to simulate nanoindentation, and the reduced modulus can be written as:

$$
\frac{1}{E_{r}}=\frac{1-v_{i}^{2}}{E_{i}}+\frac{1-v_{f}^{2}}{E_{f}}\left(1-e^{-\frac{\alpha t}{\sqrt{A_{C}}}}\right)+\frac{1-v_{s}^{2}}{E_{s}}\left(e^{-\frac{\alpha t}{\sqrt{A_{C}}}}\right)
$$

where $A_{c}$ is the contact area, $t$ is the indentation depth, $E_{s}$ and $v_{s}$ are the substrate elastic modulus and Poisson's ratio, respectively, and $\alpha$ is a numerically determined scaling parameter. One of the main problems of this model is that the Berkovich tip is a three-sided pyramid, and that the film elastic modulus is assumed to be known. Thin film Young's modulus may range with grain size and film thickness [11], thus it is difficult to know the modulus prior to film testing. An improved method using a conical tip has been suggested [5], and the reduced modulus can be written as:

$$
\frac{1}{E_{r}}=\frac{1-v_{i}^{2}}{E_{i}}+\frac{1-v_{f}^{2}}{E_{f}}\left(1-e^{-\frac{\alpha(t-h)}{\sqrt{A_{C}}}}\right)+\frac{1-v_{s}^{2}}{E_{s}}\left(e^{-\frac{\alpha(t-h)}{\sqrt{A_{C}}}}\right)
$$

where $h$ is the film thickness. Again, the film Young's modulus is not known before testing, and when the indentation depth, $t$ is equal to the film thickness, $h$, the second term disappears and the whole equation goes back to the Oliver-Pharr method.

Recently Han et. al. [9] used a numerical method to calculate the corrected contact area. In this method, the contact area is estimated by taking the measured continuous stiffness from indentation experiments and using the reduced calculated stiffness to assign the corrected contact area. 
We propose another procedure, which is a variation of the Joslin-Oliver method, and utilizes the fact that the elastic modulus does not change significantly with the indentation depth for the two gold films tested, and has the $1 / \mathrm{A}^{0.5}$ dependence, while the hardness has the $1 / A$ dependence.

\section{Experimental Details}

In order to investigate the validity of the proposed method, two gold samples were tested. One gold sample was evaporated on the mica substrate to the thickness of 200 $\mathrm{nm}$. Another sample was evaporated on the (100) silicon wafer, with a $20 \mathrm{~nm} \mathrm{Cr}$ interlayer, to the thickness of $50 \mathrm{~nm}$. The Hysitron Triboindenter was used to test the films mechanical properties. Prior to each set of tests, the tip was calibrated by indenting quartz in order to obtain the tip projected area as a function of the indentation depth for the Oliver-Pharr method. For thin films the surface roughness will strongly influence the results, so the in-situ imaging capability of the Hysitron Triboindenter was used prior to indentation (Figure 1a) to find smooth areas. The surface roughness for the $200 \mathrm{~nm}$ gold samples is below $10 \mathrm{~nm}$, and the roughness of the $50 \mathrm{~nm}$ samples is below $3 \mathrm{~nm}$. One of the advantages of the gold samples is that they do not have surface oxides, which may affect the indentation results.

\section{Results and Discussion}

Figure 2a shows the $P / S^{2}$ values for 50 and $200 \mathrm{~nm}$ gold films. The $P / S^{2}$ ratio increases with indentation depth, which proves that the substrate influences the measurements. For most homogeneous materials, other researchers have reported that $P / S^{2}$ should be a constant [5]. Reduced modulus on the other hand does not change significantly with the indentation depth (Figure $2 b$ ).

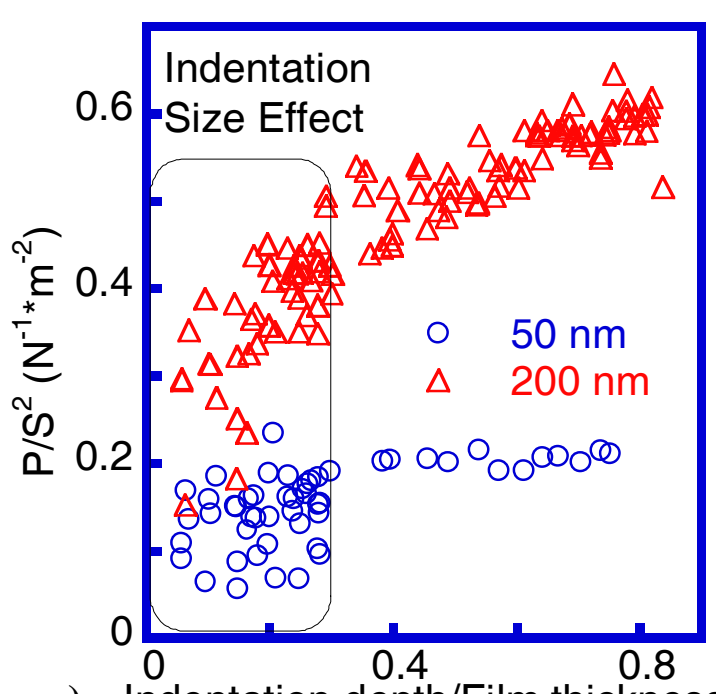

a) Indentation depth/Film thickness

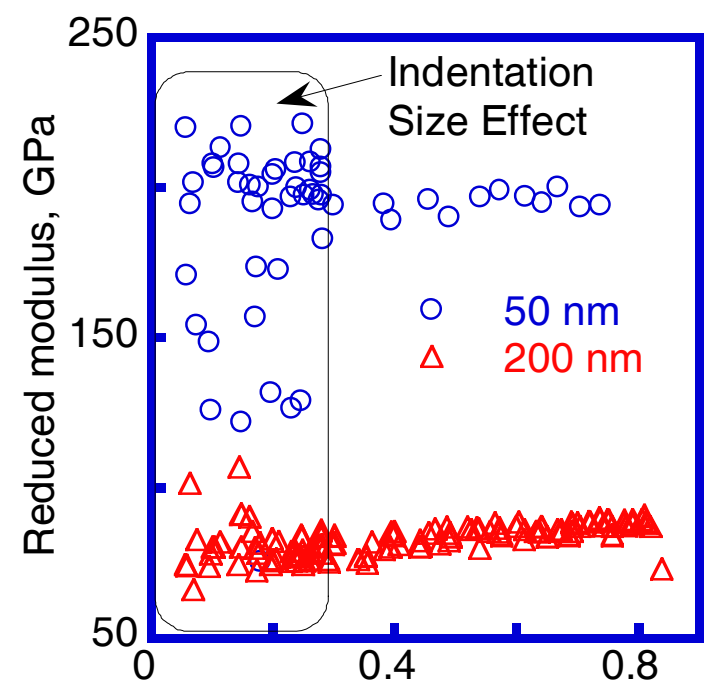

b) Indentation depth/Film thickness

Figure 2. a) $\mathrm{P} / \mathrm{S}^{2}$ and b) reduced modulus results for $200 \mathrm{~nm}$ gold on mica and $50 \mathrm{~nm}$ gold on (100) silicon wafer. 
The hardness was first calculated using the Oliver-Pharr method, which shows a steady increase with the indentation depth (Figure 3). The initial part of the experimental data, below the ratio of the indentation depth to the film thickness of 0.3 , has been explained by the indentation size and the grain size effects, which can not be corrected for using the proposed procedure.

The Joslin-Oliver method can be used to obtain the true hardness for bulk materials, while for the thin film/substrate combination, this method underestimates the substrate influence. Other researchers have shown that the elastic mismatch between the film and the substrate materials is one of the main reasons that causes incorrect hardness values [9]. We propose to reduce the elastic modulus mismatch effect on the measured thin film hardness. First the Joslin-Oliver method is used, and the data is linear fitted. For both gold samples, the shallow indentations ( $<20 \%$ of the film thickness) were excluded. The corrected hardness is:

$$
H=\frac{E_{f}}{E_{s}} \cdot \frac{h_{c}}{t} \cdot a+b
$$

where $a$ and $b$ are the linear fit parameters for the Joslin-Oliver data (Figure 3), $E_{f}$ and $E_{S}$ are the reduced moduli of the film and the substrate, respectively, $h_{c}$ is the contact depth and $t$ is the film thickness. The $200 \mathrm{~nm}$ gold film reduced modulus is $75 \mathrm{GPa}$ (Figure 2b), while the mica substrate modulus is $169 \mathrm{GPa}$. The $50 \mathrm{~nm}$ gold reduced modulus is $180 \mathrm{GPa}$ (Figure 2b), and the Cr underlayer modulus of is about $200 \mathrm{GPa}$. The proposed method results along with the Oliver-Pharr and the Joslin-Oliver hardness are shown in Figure 3 for the two gold films on mica and (100) Si substrate with a $20 \mathrm{~nm}$ Cr interlayer. When the indentation depth reaches the film thickness, the corrected hardness values approach the substrate material hardness. The proposed procedure needs to be validated further on other thin film systems.

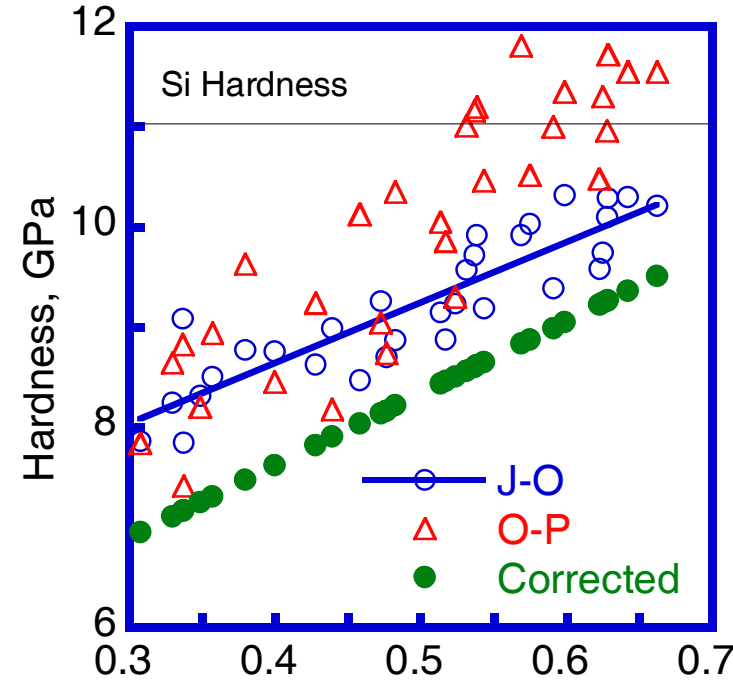

a) Indentation depth/Film thickness

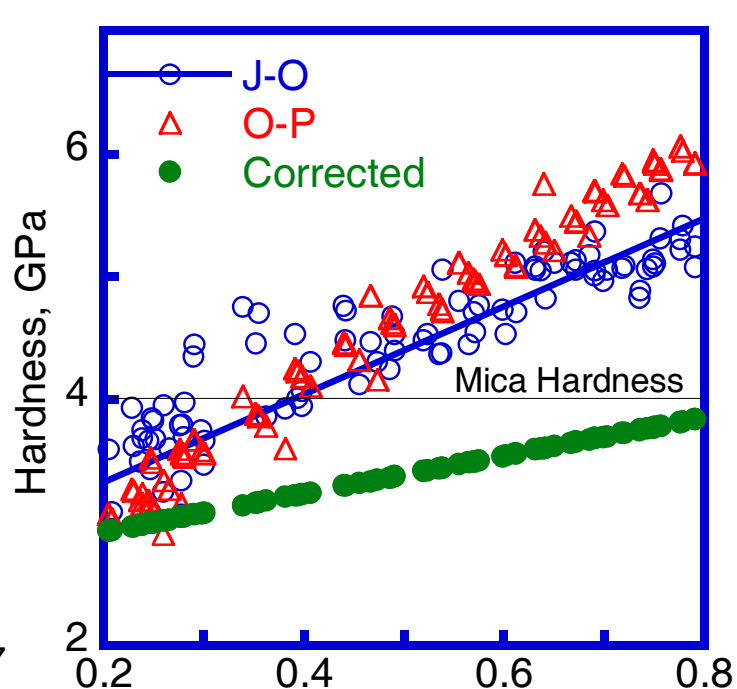

b) Indentation depth/Film thickness

Figure 3. Hardness results for a) $50 \mathrm{~nm}$ gold film on mica and b) $200 \mathrm{~nm}$ gold film on (100) Si wafer with $20 \mathrm{~nm}$ Cr interlayer. 


\section{Conclusions}

A procedure based on the Joslin-Oliver method has been proposed, and could reduce the substrate effect on hardness for ductile thin films on hard substrates if the measured film elastic modulus does not vary much with the contact depth. For the deeper indents when the indentation depth to the film thickness ratio is one, the corrected hardness values approach the substrate hardness.

\section{Acknowledgements}

The authors would like to acknowledge support for this project from NSF (CMMI-0631526 and CMMI-0600266). We would like to thank David Gracias group from John Hopkins University for providing gold sample on silicon, Piotr Cyganik from Jagiellonian University for providing gold sample on mica, and Patrick Waters from USF for help with indentation experiments.

\section{References}

1. D.T. Read and J.W. Dally, J. Mater. Res. 8 (1993) 1542.

2. T.-Y. Zhang, X. Wang, B. Huang, Mater. Sci. and Eng. A 409 (2005) 329.

3. S.P. Baker and W.D. Nix, J. Mater. Res. 9 (1994) 3131, 3145.

4. A.A. Elmastafa, D.S. Stone, Acta Mater. 50 (2002) 3641.

5. R. Saha, W.D. Nix, Acta Mater. 50 (2002) 23.

6. M. Zhao, X. Chen, Y. Xiang, J.J. Vlassak, D. Lee, N. Ogasawara, N. Chiba, Y.X. Gan, Acta Mater. 55 (2007) 6260.

7. G.M. Pharr, W.C. Oliver, and F. Brotzen, J. Mater. Res. 1 (1986) 601.

8. D.L. Joslin, W.C. Oliver, J. Mater. Res. 5 (1990) 123.

9. S.M. Han, R. Saha, W.D. Nix, Acta Mater. 54 (2006) 1571.

10. R.B. King, Int. J. Solid. Struct. 23 (1987) 1657.

11. D.E. Kramer, A.A. Volinsky, N.R. Moody and W.W. Gerberich, J. Mater. Res. 16 (2001) 3150. 